\title{
Pesquisas qualitativas em nutrição e alimentação
}

\author{
Qualitative studies in nutrition and feeding
}

Ana Maria CANESQUI

R E S U M O

O artigo revê e comenta os estudos qualitativos e os que fazem interlocução com as Ciências Sociais e Humanas na área de alimentação e nutrição. Selecionaram-se 93 artigos nas publicações registradas na base de dados do SciELO, de uma listagem geral obtida com as palavras chaves alimentação e nutrição. Classificaram-se os conteúdos em cinco assuntos: dimensões sociais, culturais, cognitivas e psicológicas da alimentação e nutrição; educação/orientação nutricional; análise e avaliação de políticas e programas de alimentação e nutrição; profissão, formação de recursos humanos e estudos teórico-metodológicos. São tecidas considerações sobre a pesquisa qualitativa nos marcos das Ciências Sociais e Humanas sobre cada um dos assuntos. Conclui-se que as pesquisas qualitativas ampliaram positivamente a interlocução da Nutrição com as Ciências Sociais e Humanas, embora requeiram aperfeiçoamento teórico-metodológico para superar os estudos descritivos, adequando o seu entendimento. Foram verificadas a forte presença da Revista de Nutrição e de periódicos das áreas de Saúde Pública/Saúde Coletiva; a concentração em alguns autores e a necessidade de apoiar a formação em Ciências Sociais e Humanas na Nutrição e incentivar a prática da multidisciplinaridade para aperfeiçoar aquelas pesquisas.

Termos de indexação: Alimentação. Antropologia. Ciências sociais. Nutrição. Pesquisa qualitativa.

A B S T R A C T

The article reviews and comments the qualitative studies and the ones that make relationship between social and human sciences in the nutrition and feeding areas; 93 articles have been selected in publications in the SciELO database, of a general listing obtained with the key words: feeding and nutrition. It was classified in five subjects: social, cultural, cognitive and psychological dimensions of feeding; education/nutritional orientation; analysis and evaluation of politics and programs of feeding and nutrition; profession, formation of human resources and theoretician and methodological studies. Considerations on qualitative research in landmarks of social sciences and commentaries on each of the subjects have been weaved. It is concluded that the qualitative research had positively extended interlocution of Nutrition with Social and Human Sciences, although requiring improvement in theories and methodologies, in some subjects, and to adjust the agreement of the qualitative research; there is a strong presence of the Revista de Nutrição and periodic of Public Health/ Collective Health in the academic production, a focus in the work of some authors and the necessity to give support in Social and Human Sciences in Nutrition formation and to improve the multidisciplinary practice in the qualitative studies.

Indexing terms: Feeding. Anthropology. Social sciences. Nutrition. Qualitative research.

\footnotetext{
1 Universidade Estadual de Campinas, Faculdade de Ciências Médicas, Departamento de Medicina Preventiva e Social. Caixa

Postal 6111, Cidade Universitária Zeferino Vaz, 13083-970, Campinas, SP, Brasil. E-mail: <anacanesqui@uol.com.br>.
} 


\section{N T R O D U Ç Ã O}

O I Fórum de Coordenadores de Cursos de Pós-Graduação em Nutrição, realizado em Salvador (BA), em 2006, apontou as necessidades de aperfeiçoar as metodologias dos estudos para alcançar maior competitividade internacional e auto-reconhecimento da área do que foi produzido'. São preocupações plausíveis para consolidar os cursos de pós-graduação, que fazem sentidos a este debate, cuja flexibilidade do tema permitiu optar pela análise do estado da arte da pesquisa qualitativa em alimentação e nutrição, registrada no sistema SciELO, no período 1985-2007.

Dos 327 resumos obtidos com as palavras-chave alimentação e nutrição 28,4\% (93) se auto-designaram como pesquisas qualitativas ou incorporaram referenciais das ciências sociais e humanas, distribuindo-se nas seguintes proporções nos periódicos identificados: Revista de Nutrição, 52,5\%; Revistas de Enfermagem, 8,5\% (Acta Paulista de Enfermagem; Revista Latino-Americana de Enfermagem e Texto e Contexto de Enfermagem); nos periódicos de Saúde Pública/Saúde Coletiva estavam 39,0\% dos resumos (Cadernos de Saúde Pública; Revista de Saúde Pública; Physis Revista de Saúde Coletiva; História Ciência, Saúde-Manguinhos; Ciência \& Saúde Coletiva e Revista Brasileira de Saúde Materno-Infantil). Entre os autores estavam: nutricionistas, enfermeiros, cientistas sociais, médicos sanitaristas ou especialistas em Saúde Coletiva/Saúde Pública, predominando os primeiros.

Pesquisas qualitativas em saúde não são novidade. Há três décadas deu-se no Brasil a interlocução das ciências sociais e humanas (Antropologia, Sociologia, Psicologia, Educação, Ciência Política) com a Saúde Pública/Saúde Coletiva e, mais recentemente, com a Nutrição.

Empregaram-se a etnografia e outras técnicas qualitativas nos clássicos estudos de comunidade, feitos por sociólogos e antropólogos no Brasil, no período de 1940 a 1970, estudando-se, além da organização social, econômica e política das populações rurais e urbanas, a produção, a distribuição e o consumo de alimentos, ao lado dos hábitos, tabus, das restrições e crenças alimentares de populações ribeirinhas, pescadoras e rurais ${ }^{2}$.

Etnografias da alimentação e nutrição, feitas por antropólogos, usaram vários instrumentos e procedimentos de obtenção de dados qualitativos inéditos sobre a família e o consumo alimentar. Foram também realizadas investigações sobre representações e práticas de consumo e sobre a reprodução da família e estratégias de sobrevivência, complementares ao Diagnóstico Nacional de Despesas Familiares (Fundação do Instituto Brasileiro de Geografia e Estatística, 1974/1975), ao lado de outros estudos antropológicos revistos ${ }^{2,3}$.

Os antropólogos estudaram, posteriormente, o simbolismo da alimentação e nutrição, as restrições e proibições alimentares, as comidas ou pratos típicos, marcadores de identidades regionais ou nacionais; as comidas rituais e religiosas, a de fast food, as dietas naturalistas, a dieta judaica; a organização e as relações sociais nas feiras e nos mercados, entre outros estudos. As análises da política de alimentação e nutrição, da profissão, da constituição do campo da nutrição e da educação alimentar fizeram interlocução com as demais ciências sociais e humanas: Sociologia, História, Ciência Política e Educação, acumulando um acervo importante de pesquisas.

Essas interlocuções ampliaram a concepção da abordagem do social nos estudos de alimentação e nutrição, antes restrita aos problemas da fome e desnutrição e aos seus determinantes, à avaliação nutricional, aos inquéritos dietéticos e às propostas de intervenção, segundo Vasconcelos ${ }^{4}$, ao estudar as publicações dos Arquivos Brasileiros de Nutrição, no período de 1944 a 1968.

Nos resumos selecionados a pesquisa qualitativa identificou-se com o uso de métodos e técnicas; com a rápida execução ou com a aproximação da realidade, dispensando amplos universos populacionais. Antes de examinar a produção científica, que indagou sobre os assuntos, con- 
ceitos, instrumentos e técnicas de coleta de informações, pergunta-se sobre o entendimento da pesquisa qualitativa, cujo debate é controvertido e polifônico, segundo as referências teóricas das ciências sociais e humanas.

Não se pretende exaurir o assunto e nem reconstituir a história da pesquisa qualitativa ou a interlocução da Nutrição com as Ciências Sociais e Humanas, mas apontar alguns aspectos do debate em torno de sua conceituação, no âmbito das Ciências Sociais e Humanas. Omissões podem ocorrer em um estudo desta natureza, advertindo-se que não foram intencionais.

\section{Consideração sobre a pesquisa qualitativa}

O qualitativo, na emergência da Antropologia da segunda metade do século XIX, identificou-se com a etnografia na inauguração dos estudos de campo das comunidades humanas, feitos por Franz Boas ${ }^{5}$ entre os esquimós, em 1880 e, anteriormente, por Morgan ${ }^{6}$ entre os iroqueses, com múltiplos desdobramentos posteriores. $\mathrm{Na}$ Antropologia Social inglesa do início do século XX a etnografia, inicialmente, descreveu as sociedades humanas de pequena-escala, orientando-se teoricamente à medida do desenvolvimento das Ciências Humanas. Ancora-se, recentemente, em novas vertentes, como a interpretativa (hermenêutica), nas revisões epistemológicas sobre o objeto da própria Antropologia e do trabalho de campo, cercado por um conjunto de problemas existenciais, morais, ideológicos políticos e éticos.

O trabalho de campo e a observação participante, nos seus primórdios, calcaram-se na objetividade do pesquisador, almejada pelos antropólogos da escola inglesa funcionalista e estrutural-funcionalista representados, respectivamente, por Malinowski ${ }^{7}$ e Radcliffe Brown ${ }^{8}$, influenciados pelas concepções das Ciências Naturais. O primeiro buscou as bases institucionais da cultura derivadas das necessidades vitais e o segundo, as leis e regularidades do funcionamento da sociedade. Retira-se a lição dos clássicos antro- pólogos que a pesquisa qualitativa não recusa a teoria e nem se esgota exclusivamente no trabalho de campo meramente descritivo.

Para outros autores, o qualitativo opõe-se ao não quantificável ${ }^{9}$, sendo que a escola norte-americana de Chicago, desde a década de 1920, incorporou a etnografia, a observação e a geração de teorias a partir dos dados empíricos. A Grounded Theory de Glaser \& Strauss ${ }^{9}$ não reduziu a importância dos clássicos estudos sobre os grupos marginais; estudantes de medicina, adoecidos crônicos, dentre outros, que, certamente, dependeram de sua profunda formação teórica e do domínio das teorias na análise das informações obtidas. Desdobramentos posteriores daquela escola muito contribuíram para aperfeiçoar as metodologias e os instrumentos da pesquisa qualitativa, lembrando-se as contribuições sociológicas de Becker $^{10}$.

A maior aceitação da pesquisa qualitativa ampliou as possibilidades de sua combinação com a pesquisa quantitativa, sempre que os objetos de estudo a reclamasse. Alguns estudos de comunidade mencionados fizeram esta combinação, enquanto os estudos dietéticos nacionais foram sempre quantitativos, predominando as pesquisas governamentais e econômicas dos orçamentos familiares e o emprego de questionários sobre o consumo, nas teses acadêmicas de nutrição, no período de 1990 a $2005^{11}$.

Outras correntes, como a etnometodo$\operatorname{logia}^{12}$ e o interacionismo simbólico ${ }^{13}$ abordam, respectivamente, o conhecimento do senso comum, valendo-se de informações e análises qualitativas, sendo a realidade social concebida como construções práticas e interativas significativas. A ação social e a intencionalidade dos indivíduos (internalidade) nos contextos e nas situações, a construção de identidades, os significados e os papéis foram abordados pelo interacionismo simbólico.

Similarmente, a fenomenologia apega-se aos indivíduos e suas interações sociais, às construções significativas da vida cotidiana, ao conhecimento do senso comum que as impregna ${ }^{14}$, 
ampliando a sociologia do conhecimento ao conhecimento da vida cotidiana, antes confinada ao conhecimento erudito. Inspirados em Alfred Schultz, aqueles autores ativaram as situações face a face e grupais, lembrando que a realidade da vida cotidiana não é espontânea, mas submetida a tipificações que são estoques de conhecimentos gerados pelo trabalho da história, ainda que possam ser negociados situacionalmente.

Estas últimas correntes enfeixam-se no que Corcuff ${ }^{15}$ designou de galáxia construtivista, que parte dos indivíduos e suas interações sociais, não sendo novas correntes teóricas à medida que, desde a década de 1960 nos Estados Unidos, Berger \& Luckmmann; Cicourrel; Goffman eram autores conhecidos das orientações mais qualitativas e interacionistas, retomadas fortemente nas ciências sociais e humanas na década de 1990 e por autores deste estudo. Estas abordagens opõem-se aos modelos de determinação estruturais, aos sistêmicos e aos funcionais. Tomam a realidade subjetivada e interiorizada pelas tipificações ou pela socialização como um duplo processo de conservação e transformação, por meio de um dos vetores - o aparelho de conversação.

Questiona-se a autoridade do autor nas abordagens antropológicas e etnográficas interpretativas e nas reflexivas que usam a história oral, as narrativas, os métodos biográficos, compreendendo os fenômenos nos seus contextos pessoal e histórico ${ }^{16,17}$.

Nas críticas pós-modernas e pós-estruturalistas o qualitativo está no cerne da desconstrução da objetividade da ciência e do pesquisador, que não é neutro, mas impregnado de valores, de ideologia, de saberes (do senso comum e erudito) que podem influenciar desde a captura e construção de seus objetos de investigação (que são e devem ser elaborados à luz dos conhecimentos científicos) às interpretações, sempre provisórias.

Denzin \& Lincoln ${ }^{18}$ afirmam, neste sentido, que não há significado fixo para ser capturado, sendo ele produto de seu tempo e espaço. Para eles, pertencentes à tradição norte-americana: "a pesquisa qualitativa é a atividade situada que traz o observador para o mundo. Ela consiste na interpretação, na prática material que torna o mundo visível... Estas práticas tomam o mundo em uma série de representações, incluindo as anotações de campo, entrevistas, conversações, fotografias, gravações e memórias do eu. Neste nível, a pesquisa qualitativa envolve as abordagens interpretativas, estuda as coisas no mundo natural, atentando para o seu sentido ou interpretação dos fenômenos em termos de significados atribuídos pelas pessoas" (tradução da autora).

Sem distinguir qualidade da quantidade, remetida ao plano das técnicas e dos procedimentos, uma visão mais abrangente, com a qual esta autora concorda, considera que "toda ciência é qualitativa à medida que estabelece uma qualidade de seus objetos de estudo no sentido de reproduzi-lo, explicá-lo ou compreendê-lo. A quantidade nada representa se não se relacionar à qualidade e os dados nunca falam sozinhos, requerem interpretação no âmbito das teorias que os alimentam, afirmando-as ou negando-as" 19 .

Briceño-León lembrou, na classificação das ciências, os estudos nomotéticos (quantitativos), que são generalizáveis porque tratam com noções, categorias que podem ser aplicadas às distintas situações, possuindo caráter universal. Os estudos idiográficos são os qualitativos: não pretendem generalização, correspondem a uma realidade histórica e esclarecem aspectos de um problema ou de um determinado grupo humano. Eles trabalham com reduzido número de informantes, selecionados mediante critérios.

Na pesquisa qualitativa a coleta de dados requer aproximação e interação entre pesquisador-pesquisado; a obtenção de informações extensivas e não apenas pontuais ou originárias de uma única fonte de coleta de dados, como as entrevistas não estruturadas, por exemplo. A análise abre-se aos conceitos e às hipóteses emergentes, que alteram o desenho inicial e provisório do estudo, buscando-se padrões de associação e não apenas descrições. Desenvolve tipologias ou explanações; voltase para os significados valorativos ou aos elementos representacionais (formas de pensamento), 
simultaneamente individuais e coletivos. Não descarta os elementos materiais envoltos nas práticas sociais, derivados da ordem social estruturadora da sociedade; os situacionais e contextuais; os simbólicos e valorativos, que podem ser específicos ou mais generalizáveis e sempre passíveis de permanências e mudanças.

A interpretação não se confunde com a repetição acrítica dos conteúdos das entrevistas, depoimentos, relatos e frases, tão freqüentemente encontrados nas pesquisas qualitativas. As entrevistas são flexíveis em relação ao número de informantes selecionados, cuja fixação numérica obedece a critérios, segundo o desenho do estudo, seu objeto, o problema formulado e as hipóteses (provisórias), os instrumentos e o quadro teórico utilizado. Nos estudos examinados o universo de informantes variou de 2, apropriado para estudo de caso, a 32, prevalecendo, em vários estudos examinados, o reduzido número de informantes, selecionado segundo sua disponibilidade ou a conveniência dos pesquisadores. Neste sentido, na seleção do número de informantes o ponto de saturação - suspensão de inclusão de novos participantes no estudo, devido à redundância e repetição das informações - é uma regra metodológica da pesquisa qualitativa que deve ser observada, não sendo apenas decisão subjetiva ou conveniência do pesquisador ${ }^{20}$.

Estas são apenas algumas definições e requisitos da pesquisa qualitativa que durante 0 século $X X$ responderam a diferentes escolas e enfoques, imersos em tradições intelectuais das Ciências Sociais e Humanas, sendo que a Clínica, a Psicologia, a Filosofia, a Epistemologia e a História também trazem aportes importantes a essas pesquisas, não consideradas nos limites desta exposição.

\section{Como a pesquisa qualitativa incorporou-se aos estudos da área de alimentação e nutrição?}

Os artigos identificados na revisão da literatura foram classificados nos seguintes assun- tos, objetos das pesquisas: (1) Dimensões sociais, culturais, cognitivas e psicológicas da alimentação e nutrição, subdivididos nos estudos sobre (1a) representações sociais; (1b) práticas, conhecimentos e comportamentos alimentares; (1c) cultura e alimentação e nutrição; (2) Educação/orientação nutricional subdivididos em (2a) gênese e constituição do campo; (2b) avaliação das intervenções educativas; (2c) análise das atuações dos profissionais (3) Análise e avaliação da política e dos programas de alimentação e nutrição: (3a) gênese e análise das políticas; (3b) avaliação dos programas de alimentação e nutrição (4) Profissão, formação de recursos humanos e campo da Nutrição; (5) Estudos teórico-metodológicos: (5a) comensalidade e mudança dos comportamentos alimentares; (5b) análises conceituais, teórico-metodológicas e desenhos de pesquisas.

Dimensões sociais, culturais, cognitivas e psicológicas da alimentação e nutrição: há estudos mais sistematizados conceitual e metodologicamente, absorvendo o conceito de representações sociais da Psicologia Social, como representações mentais da realidade ${ }^{21}$, ou como conhecimento do senso comum cotidiano ${ }^{22}$, evocando também a análise das práticas discursivas e a produção de sentido ${ }^{23}$ ou a fenomenologia, que confere importância ao conhecimento do senso comum.

Moscovici, na Psicologia Social, e Herzlich, na Sociologia, ambos ligados à escola francesa, resgataram da teoria de Durkheim, o conceito de representações coletivas definidas como categorias de pensamento social. Mocovici denominou-as de representações sociais, articulando o coletivo ao individual, embora seus críticos apontem sua permanência no individual, sendo indispensável articular ambos os níveis. Adam \& Herzlich ${ }^{24}$ admitem que, na interpretação dos fenômenos orgânicos, as pessoas se apóiam em conceitos, símbolos e estruturas interiorizadas, segundo os grupos a que pertencem, firmando-se certas doenças no imaginário coletivo. 
Há autores que tomaram as representações sociais como percepções ou concepções dos sujeitos; formas de conhecimento ou de ação, nem sempre explicitando as teorias embutidas nos conceitos que se tornam auto-evidentes para os que apenas transcreveram as falas dos entrevistados, mediante estudos descritivos e exploratórios.

Vários autores abordaram as representações $^{25-36}$. O retorno ao sujeito, a ruptura com a objetividade do conhecimento, o encontro com o vivido, com as construções sociais parecem ter motivado os pesquisadores da área de Nutrição a estudar as representações alimentares, ao lado de preocupações com as intervenções educativas impositivas e normativas desconhecedoras da complexidade das práticas alimentares e das representações sociais ${ }^{25}$, ou em função de interesses emancipatórios e ético-políticos ${ }^{37}$.

As representações da alimentação e nutrição ultrapassam as elaborações individuais e subjetivas, sendo subjetivadas, reelaboradas nas práticas, no processo de interiorização e exteriorização do que é aprendido pelos indivíduos e grupos socialmente posicionados, em um determinado tempo, nos esquemas de socialização primários e secundários, no contato com o conjunto de conhecimentos socialmente disponíveis (eruditos e do senso comum), assim como com as experiências vividas, corporais e relacionadas à saúde/doença e às regras alimentares. A alimentação e nutrição inclui o imaginário, as crenças coletivas, alteradas no tempo e no espaço, sendo indispensável à vida e à reprodução biológica e social completando-se como objeto sociocultural, econômico, histórico e político, que demanda a atenção dos pesquisadores.

Alguns textos fizeram correspondência unívoca das representações com os comportamentos e as práticas ou entre o pensar e o agir, posto pelo behaviorismo, cujas contradições e dissonâncias são difíceis de apreender somente na análise dos conteúdos das entrevistas, desafiando o pesquisador a empreender observações mais prolongadas das práticas alimentares, com- preendendo a sua impregnação por elementos valorativos, materiais, simbólicos, que são sociais, históricos e culturais, não restritos apenas à linguagem falada. Outros estudos examinados, orientados pela fenomenologia, fixaram-se nas variações das representações e dos significados, nas situações e relações sociais nos grupos observados, combinando diferentes técnicas de obtenção das informações (relatos orais, observações e entrevistas grupais).

O uso de teorias antropológicas por alguns estudos tomou as representações alimentares como modelos culturais ou do senso comum (forma de conhecimento) construído e operado na realidade ${ }^{38}$. São, portanto, os valores simbólicos e as crenças, os significados simbólicos elaborações coletivas, apresentados na forma de conhecimento, de formas de pensar e agir, integrantes da cultura e do sistema de representações simbólicas, não esgotados nas construções autônomas individuais e subjetivas.

Articulado ou não ao estudo das representações está o subitem $1 b$, sobre os conhecimentos, as atitudes e as práticas ou os comportamentos alimentares em vários autores ${ }^{39-44}$, enfocando o necessário conhecimento dos aspectos psico-sociais e motivacionais dos comportamentos alimentares individuais para explicar o seguimento ou não das dietas prescritas ou a adoção da alimentação e nutrição saudável, segundo as prescrições e concepções do saber científico nutricional. A promoção de novos comportamentos, em algumas teorias psicológicas, indica a necessidade de conhecer as crenças, as pressões sociais e os conhecimentos dos sujeitos para alterá-los, enquanto a crítica ao etnocentrismo impõe a compreensão desses elementos, como matérias culturais a ser respeitadas ou negociadas nas intervenções.

No subitem 1c os estudos manejaram conceitos antropológicos como padrões culturais, tabus, crenças, tradições, conhecimentos e significados, originários de diferentes teorias ${ }^{38,45-51}$.

Abordaram-se os modelos ou padrões culturais presidindo as escolhas alimentares, sem 
desprezar as estratégias de sobrevivência de grupos submetidos à escassez de alimentos. Tomaram-se as experiências e os significados da fome, transcendentes às condições materiais, porque ancoradas em imagens coletivas e no sistema de símbolos, socialmente produzidos ${ }^{47}$, ou os significados compartilhados grupais da fome, na leitura hermenêutica da linguagem, buscando-se as metáforas e os signos que as acompanham ${ }^{48}$, evocando-se a abordagem interdisciplinar e a presença da Antropologia na compreensão da fome crônica ${ }^{47}$, tanto quanto o seu emprego na compreensão interdisciplinar da alimentação e nutrição como fenômeno bio-cultural ${ }^{50}$.

Deslocaram-se os olhares às restrições e preferências alimentares e aos aspectos socioculturais ${ }^{49}$, cruzando-se as informações obtidas de múltiplas fontes, submetendo-as aos testes de verificação de consistência e validação das respostas, auxiliares na sua sistematização. Compararam-se os conteúdos e as múltiplas dimensões dos entendimentos do senso comum sobre algumas categorias dietéticas como o forte/fraco; quente/frio; reimoso ${ }^{51}$, obtidas dos estudos etnográficos nacionais, apontando-se a lógica das explicações, que incorporam simultaneamente conhecimentos tradicionais, reelaborações e apropriações do conhecimento dietético científico, significados e variações regionais, conteúdos simbólicos e valorativos; relações com as regras de uso do corpo, significados e conhecimentos sobre a relação corpo - alimentação e nutrição. Analisaram-se ainda quatro tipos de fontes informativas (as tribos naturalistas; os profissionais de saúde; a publicidade e a indústria) que portam representações sobre o chamado alimento natural ${ }^{52}$.

- Educação/orientação nutricional: neste item concentraram-se 14,0\% dos artigos examinados. Na análise da gênese e da constituição daquele campo (3a) abordaram-se as concepções e perspectivas de organismos internacionais ${ }^{53}$ e a objetivação do conhecimento científico produzido, como expressão de outros acontecimentos, em diferentes períodos históricos ${ }^{54}$, explorando-se a produção de conhecimento e as estratégias de intervenção ${ }^{55}$. No conhecimento acumulado nas teses defendidas em programas de pós-graduação do Rio de Janeiro (1980 a 1990) as matrizes conceituais, deslocaram-se da chamada ignorância alimentar aos estudos das representações sociais, na década de 1990, explicitando-se os discursos e os modelos fechados de significação ${ }^{37}$.

O subitem 2b abarcou um conjunto de pesquisas empíricas sobre as intervenções educativas, avaliadas pelos usuários e profissionais de saúde. Discussões sobre os modelos psicológicos de aconselhamento foram consideradas ${ }^{56}$, ao lado das tecnologias e dos conteúdos de comunicação ${ }^{57,58}$. A desatenção da orientação educacional com os aspectos cognitivos e simbólicos do comportamento alimentar apontou a necessária incorporação do método problematizador, como o reflexivo-dialético de Paulo Freire ${ }^{59}$, valorizando- o na prática da educação dialógica ${ }^{60}$.

No subitem 3c estudos empíricos sobre a atuação dos profissionais abordaram seus papéis, conhecimentos; percepções, condutas e representações em relação à orientação nutricional, sob as abordagens psico-social, fenomenológica, interacionista simbólica ou dialético-hermenêutica, sugerida por Minayo ${ }^{61}$, obtidas de entrevistas individuais ou de discussões grupais ou da pesquisa-ação, envolvendo os próprios pesquisadores no processo de intervenção, obtenção e análise das informações ${ }^{62-66}$. Identificaram-se várias dificuldades na incorporação do cuidado nutricional pelos profissionais de saúde, dentre elas: a falta de preparo e formação, os obstáculos materiais; os relacionados ao funcionamento das instituições de saúde e o excesso da demanda. Da mesma forma, alguns estudos deixaram de articular com maior precisão as teorias e a metodologia enunciadas nas análises empreendidas.

- Análise a avaliação da política de alimentação e nutrição: neste item estavam 29,0\% dos artigos selecionados. Na análise de políticas (3.a) a revisão bibliográfica de Figueiredo \& Figueiredo ${ }^{67}$ destacou as políticas de alimentação e nutrição entre as mais estudadas pelos estudos epide- 
miológicos, de avaliação nutricional, sempre quantitativos, ausentando-se, na ocasião, a análise da formulação e implementação das políticas. Avaliando-se a produção de teses de pós-graduação em Nutrição (1980-2004) sobre o assunto observou-se o deslocamento da abordagem dos processos de intervenção biológica para a sócio-política ${ }^{68}$, ou a centralidade dos intelectuais fundadores à reconstituição histórica das distintas práticas discursivas formuladoras das políticas, assim como as pesquisas avaliativas, combinadose os métodos qualitativos (análise de documentos, dos conteúdos discursivos) e as fontes quantitativas de informações.

No primeiro subitem 3a está a análise do processo político de institucionalização das políticas de alimentação e nutrição no Brasil, nos períodos de 1940 e durante os 70 destacando-se as instituições, os agentes e os diferentes programas $^{69,70}$. Reconstituiram-se o pensamento de personagens e atores formuladores relevantes, como Josué de Castro e outros ${ }^{71}$; os determinantes estruturais e conjunturais de natureza política, econômica e social na formulação e implantação das políticas de alimentação e nutrição ${ }^{72}$, estudando-se, nas diferentes conjunturas e nos vários períodos (1930-2003) as concepções, os discursos oficiais governamentais ou de atores formuladores, as mudanças nas concepções da política, os modelos de gestão, a concessão de benefícios, financiamento e relações entre Estado sociedade civil ${ }^{73}$, destacando-se os componentes temáticos da agenda de organismos internacionais comemorativos do Dia da Alimentação ${ }^{74}$.

As mudanças e as concepções da Segurança Alimentar nos planos governamentais nacionais e das organizações internacionais foram abordadas $^{75}$, propondo-se a construção da cidadania e a atuação dos nutricionistas a respeito. Nas relações entre o Estado e a sociedade civil desloca-se o enfoque das estruturas institucionais e discursivas governamentais às estratégias do movimento social de Ação da Cidadania contra a Fome, a Miséria e pela Vida ${ }^{76}$, ao lado das estratégias de combate à fome e à pobreza do programa da Comunidade Solidária ${ }^{77}$.
Na análise da política de educação alimentar e nutricional constatou-se a sua ausência na agenda da política de Segurança Alimentar ${ }^{78}$, formulando-se propostas a respeito ${ }^{79}$, da mesma forma que a inserção daquela educação nos currículos escolares do ensino fundamental, ancorada nas ações promocionais da saúde ${ }^{80}$. Assim, vale-se do espaço da escola como oportunidade de intervenção, avaliação e ampliação daquela política ${ }^{81}$. Os estudos tendem a refletir os fortes apoios da comunidade de especialistas na formulação, ampliação e na orientação daquela política, com análises que subsidiam ou refletem sobre as intervenções e propostas de promoção da saúde, centradas na alimentação e nutrição.

No subitem 3b, avaliação das políticas e programas, abordou-se o processo de descentralização do Programa de Alimentação Escolar, mediante estudo de caso e análise de desempenho do programa ${ }^{82}$. Foi também avaliado o processo de implantação, incorporando conceitos das Ciências Sociais e Humanas e as informações quantitativas e qualitativas na sua avaliação, enfocando-se as suas estruturas organizacionais, a operação dos meios e os recursos institucionais; os agentes envolvidos, o alcance das metas e dos propósitos e os julgamentos dos beneficiários ${ }^{83}$. Uchimura \& Bossi $^{84}$ avaliaram os programas de comercialização de alimentos na sua interface com a cidadania e os usos dos programas sociais ${ }^{85}$, sendo que a análise do programa Leite é Saúde mostrou sua baixa implementação ${ }^{86,87}$. Em outro tipo de pesquisa avaliativa adaptou-se de Donabedien ${ }^{88}$ o modelo de estrutura, processo e resultado, aplicado aos estudos da qualidade da atenção médica, articulando estas dimensões e não os estancando na análise de vários indicadores e resultados, reunidos em uma proposta metodológica ${ }^{89}$, usada na avaliação de vários programas da política de Segurança Alimentar, combinado múltiplas técnicas de obtenção das informações ${ }^{90,91}$.

O esforço de montagem de um desenho metodológico de avaliação dos diferentes programas foi evidente, embora permaneçam dificul- 
dades de articulação das informações qualitativas e quantitativas na avaliação de programas diferenciados. A avaliação de membros das equipes do programa de Saúde da Família concluiu a sua necessária capacitação para implantar ações de atenção nutricionais mais efetivas ${ }^{92}$.

Sobre os julgamentos dos usuários em relação aos benefícios recebidos argumentaram-se a importância de sua participação $0^{93}$ e as avaliações dos problemas nutricionais, objetos das políticas, investigando-se os sentidos da alimentação e nutrição obtida e da saúde ${ }^{94}$.

- Profissão, formação de recursos humanos e campo da nutrição: constavam neste item $15,0 \%$ dos artigos selecionados, abordando, primeiramente, a profissão e o trabalho dos nutricionistas, fazendo ou não interlocução com as Ciências Sociais e Humanas, usando-se ou criticando a teoria marxista do processo de trabalho ${ }^{95}$ ou propondo outros marcos referenciais ${ }^{96}$. Incluiram-se estudos empíricos sobre as condições e a organização do trabalho dos nutricionistas nas empresas de refeições coletivas ou na gestão dos cuidados $^{97,98}$, além de terem sido avaliados e identificados os problemas na inserção dos nutricionistas na rede básica de saúde ou nos serviços de reabilitação ${ }^{99-101}$, reconhecendo-se o pioneirismo da incipiente inserção. Processo de trabalho, subjetividade, cargas de sofrimento ou a visão funcional-administrativa das atividades orientaram conceitualmente essas pesquisas.

Em segundo lugar estavam importantes estudos históricos sobre a constituição e o desenvolvimento do campo científico da nutrição e da profissão do nutricionista ${ }^{102-106}$, ao lado das pesquisas sobre as condições e a situação de trabalho ${ }^{107}$, sempre orientadas à auto-compreensão da profissão, das diretrizes e das normas da formação dos recursos humanos, à crítica e ao equacionamento das futuras necessidades. Contextualizaram-se análises dos princípios e das diretrizes emanadas das instituições formuladoras das políticas ou das reguladoras da profissão, enquadrando-se as primeiras pesquisas na formulação e na implantação da política de formação de recursos humanos, ultrapassando a perspectiva normativa.

- Reflexões teórico-metodológicas: reuniram-se neste item $10,8 \%$ dos artigos, destacando-se as reflexões e propostas teórico-metodológicas sobre diferentes objetos, vistos na perspectiva interdisciplinar ou disciplinar (Sociologia, Antropologia, Psicologia e História da Alimentação), motivados ou não pelas intervenções. Os artigos, originários principalmente da Revista de Nutrição, revelam esforços teórico-conceituais e operacionais da pesquisa na interlocução da Nutrição com as Ciências Sociais e Humanas.

No primeiro subitem 2.1 analisam-se a comensalidade e a mudança dos comportamentos alimentares, os impactos e as resistências aos efeitos da globalização, intermediados pelas alterações do modo de vida urbano, afetando os comportamentos alimentares e o padrão de consumo ${ }^{108}$, explorando-se um conjunto de determinantes (materiais, culturais e subjetivos). Critica-se a massificação e a importação de modelos culturais hegemônicos de padrões alimentares ${ }^{109}$, que deslocam o sistema de significação. Analisamse as mudanças dos comportamentos alimentares - o caso francês ${ }^{110}$, sintonizando as alterações na cultura alimentar com os processos de mudança econômica, sem observar as possíveis divergências e resistências. Tomam-se os modelos de consumo e seus fatores determinantes expressos nos hábitos alimentares ${ }^{111}$, usando-se o conceito de sistemas alimentares na perspectiva histórica e comparativa, que incorpora também os diferentes agentes sociais e suas relações (produtores, distribuidores, consumidores e Estado) na compreensão, construção e na mudança dos hábitos alimentares, que não gozam de autonomia em relação aos fatores determinantes, deixando de ser apenas motivações comportamentais.

Propostas teóricas metodológicas e de desenhos de investigação (item $2 b$ ) nas reflexões sociológicas de Poulain \& Proença ${ }^{112}$ abraçam a pluridisciplinaridade sobre o ato alimentar, como objeto biocultural, situado nas inter-relações entre um agrupamento social e seu meio. O conceito 
de espaço social alimentar abre-se às múltiplas dimensões: o comestível, a produção alimentar, o culinário, os hábitos de consumo, a temporalidade e as diferenças.

A alimentação e nutrição como fato bio-cultural conclama a contribuição das Ciências Sociais e Humanas com a biomedicina e a Nutrição, tomando-se a dimensão sociocultural e as relações envoltas nas práticas alimentares de mulheres durante o ciclo gravídico-puerperal, que se prestam simultaneamente à ciência e à cultura, expondo-se as mulheres às regras advindas de saberes diversificados, oriundos das culturas do grupo família, de gênero e da biomedicina. Reforça-se a insistente crítica da Antropologia às posturas etnocêntricas de não tomar como inadequados ou como ignorância os elementos culturais e simbólicos da alimentação e nutrição 50,51,113

$\mathrm{Na}$ análise das teorias que ancoram as mudanças de atitudes, crenças e percepções dos comportamentos, Assis \& Nahas ${ }^{113}$ apontam as seguintes teorias: cognitiva; do autocontrole; health belief model; os modelos de prevenção da recaída, transteórico e integrativos, abraçando estes últimos, que consideram os planos organizacional, ambiental e individual no estudo e nas orientações das mudanças dos comportamentos alimentares $^{114}$

A reflexão sobre o uso do conceito de representações, sua flexibilidade, suas alterações no tempo e suas interferências nos relatos da alimentação e nutrição obtida, conduziu Garcia ${ }^{115}$ a examinar os conteúdos dos relatos, as perguntas formuladas e as respostas obtidas, as imprecisões, flutuações, indução nas respostas, hesitações do entrevistado e outras questões, que reforçam o emprego dos instrumentos da pesquisa qualitativa e daquele conceito, contrariando a objetividade dos inquéritos alimentares.

O conceito de risco, emprestado da Epidemiologia, ancorado na probabilidade matemática, relativiza-se para Arnaiz ${ }^{116}$ como construção social, que não despreza os sujeitos, o sistema de significação e a cultura na sua geração, cuja análise envolve a perspectiva socioantropológica e quali- tativa para compreender as diversidades de pontos de vista sobre a aplicação das biotecnologias, o impacto e as atitudes diante dos alimentos transgênicos. Poulain \& Proença ${ }^{117}$ discutem à luz da Sociologia e da Antropologia o conceito de práticas alimentares, nas diferentes teorias e múltiplas dimensões que o encerram, desenhando metodologia e instrumentos para o seu estudo, mediante cuidadoso emprego dos instrumentos e operacionalização daquele conceito e de suas dimensões implicadas.

\section{O N CLUS Ã O}

Devido à forma desta exposição, centrada em cada item pesquisado, exime-se de retomá-las nestas conclusões, reconhecendo-se a importância das contribuições feitas ao desenvolvimento e à legitimação da pesquisa qualitativa em alimentações e nutrição. Em função de alguns problemas apontados indaga-se sobre o seu aperfeiçoamento, traçando-se algumas sugestões bastante gerais.

Decorrente do reducionismo do entendimento deste tipo de pesquisa, aos métodos e instrumentos ou à sua rapidez, há que ampliar as concepções e as articular às teorias para que não permaneçam nas descrições e repetições das falas dos entrevistados, que, certamente, ultrapassaram-se por autores que manejam os conceitos das Ciências Sociais e Humanas. Entretanto deve-se reforçar a necessidade de permanente capacitação dos pesquisadores neste tipo de pesquisa, nos seus pressupostos, nas teorias, no desenho, nas técnicas, nos instrumentos e nos modelos de análise, aplicados também às análises históricas e das políticas.

Os estudos foram mais divulgados por algumas revistas, como a de Nutrição, seguida pelos periódicos das áreas de Saúde Pública/Saúde Coletiva e, por último, pelas revistas de Enfermagem, demonstrando a abertura de suas políticas editoriais à pesquisa qualitativa e não apenas aos estudos quantitativos, embora se aponte a necessidade de qualificar outras revistas de Nutrição, 
para maior fluência da produção acadêmica, em especial, dos cursos de pós-graduação.

Neste estudo verificou-se a concentração de publicações em alguns autores, com tendência à diversificação e ao compartilhamento da autoria, que são bastante desejáveis à capacitação de alunos de pós-graduação. A interlocução dos estudos com as abordagens das Ciências Sociais e Humanas, que presidem os estudos qualitativos, impõe a capacitação e a formação permanente de atuais e futuros pesquisadores. Ainda que os currículos de graduação em Nutrição incorporem aquelas ciências, sensibilizando futuros profissionais, a aproximação tem sido mais disciplinar do que multidisciplinar e associada à formação básica ${ }^{118}$, problema a ser equacionado também nos cursos de pós-graduação, que poderá apoiar as pesquisas qualitativas futuras.

A inter-relação entre as Ciências Sociais e Humanas e a Nutrição é viável, mas seus pontos de partida são distintos. As primeiras são compreensivas, históricas e ideológicas, implicam na subjetivação e na intersubjetividade, sendo relacionais e não apenas restritas à subjetividade dos indivíduos, embora esta dimensão não se exclua. As segundas ancoram-se na biologia, na fisiologia e sua verdadeira inter-relação requer mais do que a transferência de técnicas e dos procedimentos de investigação, sendo que a recomposição das disciplinas pode gerar novas formas de pesquisa, na produção de objetos híbridos, suscitando dos pesquisadores o domínio e o manejo das teorias, metodologias e dos instrumentos adotados pelas disciplinas, entrecruzadas na prática multi ou interdisciplinar. Nos estudos examinados deparou-se com esforços nesse sentido, contidos especialmente nas reflexões conceituais e nas propostas de desenho de pesquisas multidisciplinares, que, certamente, terão impactos positivos na interlocução das Ciências Sociais e Humanas com a Nutrição.

Afirma-se que o século $X X$ foi o das disciplinas, como poderosas entidades especializadas, profissionalizadas e fortemente departamentais. A definição dos métodos das ciências sociais e humanas, experimentada especialmente na segunda metade do século XX, favoreceu o fortalecimento da interdisciplinaridade. O século XXI abre maiores flancos ao trabalho interdisciplinar, no qual as fronteiras das disciplinas tornam-se permeáveis. As estratégias pessoais, institucionais e no plano do conhecimento são necessárias a este tipo de trabalho, selecionando-se tópicos que conclamem múltiplos olhares. São pesquisas operacionais e estratégicas, diferentes das básicas e produtoras de conhecimento ${ }^{62}$. Embora voltadas à solução de problemas, elas se nutrem das teorias produzidas, sendo a multi e a pluridisciplinaridade férteis nestes territórios de colaboração mútua de especialistas, não subordinando um conhecimento ou disciplina a outra, nutrindo-se mutuamente e ultrapassando os vários obstáculos institucionais, corporativos e relacionais na investigação de objetos híbridos.

Deu-se positivamente a compreensão da complexidade do fato alimentar nas suas dimensões valorativas, simbólicas, subjetivas e motivacionais, além das fisiológicas e materiais, assim como a assimilação de conceitos e de metodologias das Ciências Sociais e Humanas nas análises da ampla temática abordada e mapeada neste texto. Isso requer de parcela dos estudos empíricos o aperfeiçoamento, o uso coerente das teorias e a maior sintonia de certas abordagens enunciadas nas metodologias com os resultados das análises obtidas. A incorporação e a atualização de conceitos e abordagens teóricos-metodológicos, que tomam as políticas ou a história, são desejáveis para aquelas aproximações mais descritivas, não extensivas ao conjunto da produção examinada.

\section{REFERÊ NCIAS}

1. Kac G, Fialho E, Santos SMC. Reflexões do I Fórum de coordenadores de programa de pós-graduação em nutrição no Brasil. Rev Nutr. 2006; 19(6): 785-92.

2. Canesqui AM. Antropologia e alimentação. Rev Saúde Pública.1988; 22(3):207-16.

3. Canesqui AM. Comentários sobre os estudos antropológicos da alimentação. In: Canesqui AM, 
Garcia RWD, organizadores. Antropologia e nutrição: um diálogo possível. Rio de Janeiro: Editora Fiocruz; 2005. p.23-47.

4. Vasconcelos FAG. Os arquivos brasileiros de nutrição: uma revisão científica em nutrição no Brasil (1944-1968). Cad Saúde Pública. 1999, 15(2):303-16.

5. Boas F. The Central Eskimo. Washington (DC); 1888

6. Morgan LH. League of Iroquois [reimpresso]. New Haven; 1954,

7. Malinowski B. Uma teoria científica da cultura. Rio de Janeiro. Zahar Editores; 1975.

8. Radcliffe-Brown AR. Estrutura e função na sociedade primitiva. Petrópolis: Vozes; 1973.

9. Glaser BG, Strauss A. The discovery of grounded theory. Chicago: Aldir Publishing; 1967.

10. Becker HS. Métodos de pesquisa em ciências sociais. São Paulo: Hucitec; 1997.

11. Vasconcelos FAG. Tendências históricas dos estudos dietéticos no Brasil. Hist Ciênc Saúde-Manguinhos. 2007; 14(4):1341-56.

12. Goffman I. The presentation of self in everyday life. New York: Doubleday; 1959.

13. Garfinkel H. Studies in ethnomethodology. New Jersey: Prentice Hall; 1967.

14. Berger P, Luckmann T. A construção social da realidade. 7a.ed. Petrópolis: Vozes; 1987.

15. Corcuff P. A nova sociologia: construções da realidade. Bauru: Edusc; 2001.

16. Chamberlangne $P$, Bornmat J, Wengraf $T$. The Turn of biographical method in social science. Comparative Issues Examples. London: Routledge; 2000.

17. Roberts B. Biographical research. Buckinghan: Open University Press; 2002.

18. Denzin NK, Lincoln Y. The discipline and practice of qualitative research. In: Denzin NK, Lincoln KS. Handbook of qualitative research. 2nd ed. London: Sage Publications; 2000.

19. Briceño-León R. Quatro modelos de integração de técnicas qualitativas e quantitativas de investigação nas ciências sociais. In: Goldenberg P, Marsiglia RGM, Gomes MA, organizadores. O clássico e o novo: tendências, objetos e abordagens em ciências sociais e saúde. Rio de Janeiro: Editora Fiocruz; 2003. p.157-83.

20. Fontarella BJB, Ricas J, Turato ER. Amostragem por saturação nas proposições qualitativas em saúde: contribuições teóricas. Cad Saúde Pública. 2008; 24(1):17-27.

21. Moscovici S. Representação social na psicanálise. Rio de Janeiro: Zahar;1978.
22. Leme MAVS. O impacto da teoria das representações sociais. In: Spink MJ, organizador. O conhecimento no cotidiano: as representações sociais na perspectiva da psicologia social. São Paulo: Brasiliense; 1993. p.46-57.

23. Spink MJ, Freeze RM. Práticas discursivas e produção de sentidos a perspectiva da Psicologia Social. In: Spink MJ, organizador. Práticas discursivas e produção de sentidos no cotidiano: aproximações teóricas e metodológicas. São Paulo: Cortez; 1998. p:41-60.

24. Adam P, Herzlich C. Sociologia das doenças e da medicina. Bauru: Edusc; 2001.

25. Garcia RWD. Representações sociais da alimentação e saúde e suas repercussões no comportamento alimentar. Physis Rev Saúde Coletiva. 1997; 7(2):51-68.

26. Garcia RWD. Práticas e comportamento alimentar no meio urbano: um estudo no centro da cidade de São Paulo. Cad Saúde Pública. 1997; 13(2): 455-67.

27. Garcia RWD. A dieta hospitalar na perspectiva dos sujeitos envolvidos em sua produção e planejamento. Rev Nutr. 2006; 19(1):93-101.

28. Ciaponi MH, Tonete VCP, Pellengill MAM, Chebaci RYS. Representações da equipe de enfermagem sobre a criança desnutrida e sua família. Rev LatinoAm Enfermagem. 1999; 17(3):17-24.

29. Silva DO, Racine EGIG, Queiroz FO. Concepções de profissionais de atenção básica sobre alimentação saudável no Distrito Federal, Brasil. Cad Saúde Pública. 2002; 18(5):1367-77.

30. Santos LAS. Uso e percepções da alimentação alternativa no estado da Bahia: um estudo preliminar. Rev Nutr. 2001; 14(Supl 0):35-40.

31. Barbosa JAG, Freitas MIF. Representações sociais sobre a alimentação por sonda obtida de pacientes adultos hospitalizados. Rev Latino-Am Enfermagem. 2005; 13(2):235-42.

32. Frota MA, Barroso MGT. Repercussão da desnutrição infantil na família. Rev Latino-Am Enfermagem. 2005; 13(6):996-1000.

33. Bandoni DH, Brasil BG, Jaime PC. Programa de alimentação do trabalhador: representações sociais de gestores locais. Rev Saúde Pública. 2006; 40(5):237-42.

34. Gerhardt TE. Situação de vida, pobreza e saúde: estratégias alimentares e práticas sociais no meio urbano. Cien Saúde Coletiva. 2003; 8(3):713-26.

35. Grando LH, Rolin MA. Os transtornos da alimentação sob a ótica dos profissionais de enfermagem. Acta Paulista de Enfermagem. 2006; 19(3):265-70.

36. Grando LH, Rolin MA. Família e transtornos alimentares: as representações dos profissionais de 
enfermagem de uma instituição universitária de atenção à saúde mental. Rev Latino Am Enfermagem. 2005; 13(6):989-95.

37. Lima ES, Oliveira CS, Gomes MCR. Educação nutricional: da ignorância alimentar à representação social na pós-graduação do Rio de Janeiro (1980-1998). Hist Cien Saúde-Manguinhos. 2003; 10(2):602-35.

38. Assis AMO, Freitas MCS, Oliveira TC, Prado MS, Sampaio LR, Machado AD, et al. Bró, caxixe e ouricuri: estratégias de sobrevivência no semi-árido baiano. Rev Nutr. 1999; 12(2):159-66.

39. Alves HJ, Boog MCF. Comportamento alimentar em moradia estudantil: um espaço para a promoção da saúde. Rev Saúde Pública. 2007; 41(2): 197-204.

40. Perez DS, Franco LJ, Santos MA. Comportamento Alimentar de mulheres portadoras do diabetes tipo 2. Rev Saúde Pública. 2006; 40(2):310-17.

41. Souto S, Furo-Bucher JSN. Práticas indiscriminadas de dietas de emagrecimento e o desenvolvimento dos transtornos alimentares. Rev Nutr. 2006; 19(6): 693-704.

42. Rotemberg S, de Vargas S. Práticas alimentares e o cuidado da saúde: da alimentação da criança à alimentação da família. Rev Bras Saúde Mater Inf. 2004; 4(1): 85-94.

43. Cavalcanti APR, Dias MR, Rodrigues CFF, Gouveia CNNA, Ramos DD, et al. Crenças e influências sobre dietas de emagrecimento entre obesos de baixa renda. Cien Saúde Coletiva. 2007; 12(6): 1567-74.

44. Santele O, Lefevre AMC, Cervato AM. Alimentação e suas representações sociais entre moradores de instituição de longa permanência para idosos em São Paulo, Brasil. Cad Saúde Pública. 2007; 23(12): 3061-5.

45. Vitolo MR, Patin RV, Von Bulow AC, Ganzerli M, Fisberg M. Conhecimentos e crendices populares de puérperas na prática da amamentação. Rev Nutr. 1994; 7(2):132-47

46. Trigo M, Roncada MJ, Stewien GTM, Pereira IMTB. Tabus alimentares em região norte do Brasil. Rev Saúde Pública. 1989; 23(6):455-64.

47. Freitas MO. Uma abordagem fenomenológica da fome. Rev Nutr. 2002; 15(1):53-69.

48. Freitas MCAS, Pena PGL. Segurança alimentar e nutricional: a produção de conhecimento com ênfase nos aspectos da cultura. Rev Nutr. 2007; 20(1):69-81.

49. Costa-Neto EM. Restrições e preferências alimentares em comunidades de pescadores do Município de Conde, Estado da Bahia, Brasil. Rev Nutr. 2000; 13(2):117-26.
50. Baião MR, Deslandes SF. Alimentação na gestação e puerpério. Rev Nutr. 2006; 19(2):245-53.

51. Canesqui AM. A qualidade dos alimentos: análise de algumas categorias da dietética popular. Rev Nutr. 2007; 20(2):203-16.

52. Lifschitz J. Alimentação e cultura: em torno do natural. Rev Physis Saúde Coletiva. 1997; 7(2):69-83.

53. Boog MCF. Educação nutricional: presente, passado e futuro. Rev Nutr. 1997; 10(1):5-19.

54. Lima ES. Gênese e constituição da educação alimentar: uma síntese. Physis Rev Saúde Coletiva. 1997; 7(2):9-29.

55. Lima ES. Gênese e desenvolvimento da educação alimentar: a instauração da norma. História Cienc Saúde-Manguinhos. 1998; 5(1):57-84

56. Rodrigues EM, Soares FPT, Boog MCF. Resgate do conceito de aconselhamento no contexto do atendimento nutricional. Rev Nutr. 2005; 18(1):119-28.

57. Boog MCF, Vieira CM, Oliveira NL, Fonseca O, L'Abbate S. Utilização de vídeo como estratégia de educação nutricional para adolescentes: comer.. o fruto ou o produto? Rev Nutr. 2003; 16(3): 281-93.

58. Frota MA, Albuquerque CM, Linard AG. Educação popular em saúde no cuidado à criança desnutrida. Texto Contexto Enf. 2007; 1692):246-53.

59. Freire P. Pedagogia da autonomia: saberes necessários à prática educativa. São Paulo: Paz e Terra; 2000.

60. Rodrigues EM, Boog MCF. Problematização como estratégia de educação nutricional com adolescentes obesos. Cad Saúde Pública. 2006; 22(5): 923-31.

61. Minayo MCS. O desafio do conhecimento: pesquisa qualitativa em saúde. São Paulo: Hucitec 1995.

62. Boog MCF. Dificuldades encontradas por médicos e enfermeiros na abordagem dos problemas alimentares. Rev Nutr.1999; 12(3):251-72.

63. Campos SH, Boog MCF. Cuidado nutricional na visão de enfermeiras docentes. Rev Nutr. 2006; 19(2):145-55.

64. Bogus CM, Mogueira-Martins MCF, Morae DEB, Taddei JACC. Cuidados oferecidos pelas creches: percepções de mães e educadoras. Rev Nutr. 2007; 20(5):499-514

65. Schtichting S, Boog MCF. Lunchtime as a therapeutic moment: a health education approach with alcohol-dependent women. Rev Latino-Am Enfermagem. 2007; 15(9):350-84.

66. Boog MCF. Educação nutricional em serviços públicos de saúde. Cad Saúde Pública. 1999; 15(Supl 2):139-47. 
67. Figueiredo M, Figueiredo A. A avaliação política e avaliação de políticas; um quadro de referência teórica. An Conj. 1986; 1(3):15-25.

68. Henrique FCS, Lira PIC, Santos SMC, Andrade SLLS. Tendências do campo de avaliação de intervenções públicas de alimentação e nutrição em programas de pós-graduação no Brasil: 1980-2004. Cad Saúde Pública. 2007; 23(1):2972-81.

69. L’Abbate S. As políticas de alimentação e nutrição no Brasil. I. Período de 1940 a 1964. Rev Nutr Puccamp. 1988; 1(2):87-138.

70. L'Abbate S. As políticas de alimentação e nutrição. II. A partir dos anos setentas. Rev Nutr Puccamp. 1989; 2(1):7-54.

71. Arruda BKG. A Geografia da fome: da lógica regional à universalidade. Cad Saúde Pública. 1997; 13(8):545-9.

72. Arruda BKG, Arruda IKG. Marcos referenciais da trajetória das políticas de alimentação e nutrição no Brasil. Rev Bras Saúde Mater Inf. 2007; 7(3): 319-26.

73. Vasconcelos FAC. Combate à fome no Brasil: uma análise histórica de Vargas a Lula. Rev Nutr. 2005; 1(18):439-57.

74. Coelho AIM, Campos MTFS, Lopes MLM, Novaes JR. O Dia mundial da alimentação: duas décadas no combate a problema da alimentação mundial. Rev Nutr. 2005; 18(3):401-18.

75. Valente FLS. O Combate à fome e a segurança alimentar e nutricional: o direito à alimentação adequada. Rev Nutr. 1999; 10(1): 20-36.

76. Santos LA. Educação alimentar e nutricional no contexto da promoção de práticas alimentares saudáveis. Rev Nutr. 2005; 18(5):681-92.

77. Ferreira V, Magalhães R. Nutrição e promoção da saúde: perspectivas atuais. Cad Saúde Pública. 2007; 23(7):1674-81.

78. Vasconcelos FAG. Fome, solidariedade e ética: uma análise do discurso da ação da cidadania contra fome, a miséria e pela vida. Hist Cienc Saúde-Manguinhos. 2004; 11(2):259-77.

79. Burlandy L, Laha ME. Redes de política no combate à fome e à pobreza: a estratégia da comunidade solidária no Brasil. Cienc Saúde Coletiva. 2007; 12(6):1543-52

80. Bizzo MLG, Leder L. Educação nutricional nos parâmetros curriculares para o ensino fundamental. Rev Nutr. 2005; 18(5):661-7.

81. Cyrino EG, Pereira MLT. Reflexões sobre uma proposta de integração saúde-escola: o projeto saúde e educação de Botucatu. Cad Saúde Pública. 1999; 15(Supl.2):39-44.
82. Spinelli MAS, Canesqui AM. O Programa de alimentação escolar: da centralização à descentralização. Rev Nutr. 2002; 15(1):105-17.

83. Spinelli MAS, Canesqui AM. Descentralização do programa de alimentação escolar em Cuiabá: 1993-1996. Rev Nutr. 2004; 17(2):151-65.

84. Uchimura KL, Bossi MLM. Programa de comercialização de alimentos: uma análise da modalidade de intervenção em interface com a cidadania. Rev Nutr. 2003; 16(4):387-97.

85. Uchimura KY, Bosi ML. O mercado dos pobres: um enfoque qualitativo da utilização de programas sociais de alimentação. Cad Saúde Pública. 2004; 20(2):482-91.

86. Santana LAA, Santos SMC. Sistema de vigilância alimentar e nutricional na implementação do programa leite é saúde: avaliação dos municípios baianos. Rev Nutr. 2004; 17(3):283-90.

87. Pereira G, Castro IRR. Considerações sobre o plano de combate à fome e à miséria. Cad Saúde Pública. 1993; 9(1):106-13.

88. Donabedien. A the seven pillars of quality. Arch Pathol Lab Med. 1990; 114:115-8.

89. Santos SMC, Santos LMP. Avaliação de políticas públicas de segurança alimentar de combate à fome no período 1995-2002. 1. abordagem metodológica. Cad Saúde Pública. 2007; 23(5):1029-40.

90. Santos LMP, et al. Avaliação de política de segurança alimentar de combate à fome no período 1995-2002: 2. programa de alimentação do trabaIhador. Cad Saúde Pública. 2007; 23(8): 1931-45.

91. Martins MC, Santos LMP, Santos SMC, Santos LMP, Araújo MLN, Martins MC, et al. Avaliação de políticas públicas de segurança alimentar de combate à fome. programa das ações de combate às deficiências de vitamina"A". Cad Saúde Pública. 2007; 23(9):2081-93.

92. Fernadez PMF, Voci SM, Kamata LM, Najas MS, Souza ALM. Programa de saúde da família e ações em nutrição em um distrito de saúde do município de São Paulo. Ciên Saúde Coletiva. 2005; 10(3): 747-55.

93. Machado MFAS. Vieira NFC. Participação na perspectiva de mães de crianças desnutridas. Rev Latino-Am Enfermagem. 2004; 12(1):76-82.

94. Stolte DH, Henington EA, Bernardes JS. Sentidos da alimentação e da saúde:contribuição para a análise do programa de alimentação ao trabalhador. Cad Saúde Pública. 2006; 22(9):1915-24.

95. Viana SV. Perspectiva relacional no estudo do processo de trabalho em saúde: contribuição para uma nova abordagem a partir do estudo da prática do nutricionista no campo da alimentação institucional. Cad Saúde Pública. 1995; 4(11):616-20. 
96. Bossi MLM. Trabalho e subjetividade: cargas de sofrimento na prática de nutrição social. Rev Nutr. 2000; 13(2):107-15.

97. Analoni JA. Situação de trabalho dos nutricionistas em empresas de refeição coletiva de Minas Gerais: trabalho técnico, supervisão ou gerência. Rev Nutr. 1999;12(3):241-60.

98. Matos $\mathrm{CH}$, Proença RPC. Condições de trabalho e estado nutricional de operadores do sistema de alimentação coletiva: um estudo de caso. Rev Nutr. 2003; 16(4):493-502.

99. Souza AA. Proença RPC. Tecnologias de gestão dos cuidados nutricionais: recomendações para qualificação do atendimento nas unidades de alimentação e nutrição. Rev Nutr. 2004;17(4): 425-36.

100. Oliveira TRPR, Radicchi AL. A inserção do nutricionista na equipe de atendimento ao paciente em reabilitação física e funcional. Rev Nutr. 2005; 18(5):601-11.

101. Pádua JG, Boog MCF. Avaliação da inserção do nutricionista na rede básica de saúde dos municípios da região metropolitana de Campinas. Rev Nutr. 2006; 19(4):413-24.

102. Vasconcelos FAC. Fome e eugenia na constituição do campo da nutrição em Pernambuco: uma análise de Gilberto Freyre, Josué de Castro e Nelson Chaves. Hist Cienc Saúde-Manguinhos. 2001; 8(2):315-39.

103. Vasconcelos FAG. Origem e conformação do campo de nutrição em saúde pública em Pernambuco: uma análise histórico-estrutural. Rev Nutr. 2001; 14:(Supl.)13-20.

104. Vasconcelos FMG. O nutricionista no Brasil: uma análise histórica. Rev Nutr. 2002;15(2):127-38.

105. Coelho HAL. Formação do profissional nutricionista na América Latina e no Brasil: com ênfase em Pernambuco. Aliment Nutr. 1983; 4(11):47-51.

106. Costa NMSC. Revisitando os estudos e eventos sobre a formação do nutricionista no Brasil. Rev Nutr. 1999;12(1):5-74.

107. Rotemberg S. Prado SD. Nutricionistas: quem somos? Rev Nutr Puccamp. 1991; 4(1-2):41-64.
108. Garcia RWD. Reflexos da globalização na cultura alimentar: considerações sobre as mudanças na alimentação urbana. Rev Nutr. 2003; 16(6):483-92.

109. Andrade A, Bossi MLM. Mídia e subjetividade: impacto no comportamento alimentar feminino. Rev Nutr. 2003; 16(1):117-25.

110. Lambert JL, Batalha MO, Stresser RL. Silva AL, Luchese T. As principais evoluções dos comportamentos alimentares: o caso da França. Rev Nutr. 2005; 18(5):577-91.

111. Oliveira SP, Thebaud-Mondy A, Assis MAA. Estudo do consumo alimentar: em busca de uma abordagem multidisciplinar. Rev Saúde Pública. 1997; 31(2):201-8.

112. Poulain JP, Proença RPC. O espaço social alimentar: um instrumento para o estudo dos modelos alimentares. Rev Nutr. 2003; 16(3):245-56.

113. Freitas MCS. Educação Nutricional: aspectos sócio-culturais. Rev Nutr. 1997; 10(1):45-9.

114. Assis MAA, Nahas MV. Aspectos motivacionais em programas de mudanças do comportamento alimentar. Rev Nutr. 1999; 12(1):33-41.

115. Garcia RWD. Representações sobre consumo alimentar e suas implicações em inquéritos alimentares: estudo qualitativo em sujeitos submetidos à prescrição dietética. Rev Nutr. 2004 17(1):15-28.

116. Arnaiz MG. Pensando sobre el riesco alimentario y su aceptabilidad: el caso de los alimentos transgênicos. Rev Nutr. 2004; 17(2):125-49.

117. Poulain JP, Proença RPC. Reflexões metodológicas para o estudo das práticas alimentares. Rev Nutr. 2003; 16(4):365-86.

118. Canesqui AM, Garcia RWD. Ciências sociais e humanas nos cursos de nutrição. In: Canesqui AM, Garcia RWD. Antropologia e nutrição: um diálogo possível. Rio de Janeiro: Editora Fiocruz; 2005. p.255-74

Recebido em 3/4/2008 Aprovado em: 9/12/2008 
